\title{
False Positives and the Detection of Cyclodextrin Inclusion Complexes by Electrospray Mass Spectrometry
}

\author{
John B. Cunniff and Paul Vouros \\ Department of Chemistry and Barnett Institute of Chemical Analysis, Northeastern University, Boston, \\ Massachusetts, USA
}

\begin{abstract}
The results of previous works that have claimed to detect cyclodextrin inclusion complexes via the "soft" ionization technique of electrospray ionization mass spectrometry are revisited. A more extensive study of cyclodextrin mixtures with amino acids and small peptides demonstrates that amino acid and peptide "complexes" are detected by electrospray mass spectrometry regardless of the presence (or not) of an aromatic moiety on the side chain. Amino acids that may be least likely to form hydrophobic inclusion complexes with cyclodextrin in solution generally show the most intense complex ions. The data suggest that these "complexes" are, in all likelihood, electrostatic adducts formed during the electrospray process. Systematic controls are suggested to ensure that "false positives" do not negate many of the claims concerning the detection of solution-derived noncovalent compounds. (J Am Soc Mass Spectrom 1995, 6, 437-447)
\end{abstract}

\begin{abstract}
$\Gamma_{\beta-c y c t o}$ yclodextrins are cyclic oligosaccharides composed of six $(\alpha-)$, seven $(\beta-)$, or eight $(\gamma)$ glu$\beta$-cyclodextrin). Dissolved in water, the hydroxyl groups arrange on the outer surface (or outer rim) of the ring, which results in an internal cavity that is slightly hydrophobic. This inner cavity may "host" a molecule that is less polar than water provided the two are compatible in terms of size and shape. The "guest" is oriented in the cyclodextrin cavity such that contact between the hydrophobic parts of the guest and cavity is maximized [1]. In turn, hydrophilic parts of the guest molecule remain as far outside the complex cavity as possible to maximize contact with the polar solvent. These complexes include proteins and amino acids that contain aromatic side chains [i.e., phenylalanine (Phe), tyrosine (Tyr), and tryptophan (Try)] [2]. Recently, cyclodextrins have been used in the separation of dansylated amino acids by micellar electrokinetic capillary chromatography [3] and with the chiral separation of dansylated amino acids by capillary zone electrophoresis (CZE) [4]. A naphthyl group, which is introduced into the amino acid via dansylation, is responsible for its complexation with the cyclodextrin.
\end{abstract}

Address reprint requests to Dr. Paul Vouros, 101 Hurtig Hall, Northeastern University, Boston, MA 02115.
After the detection of noncovalent receptor-ligand complexes by electrospray ionization (ESI) mass spectrometry was first reported by Ganem et al. $[5,6]$ in 1991, an assiduous pursuit of noncovalent complexes followed within the mass spectrometry community. Within the last two years, it has been reported that the formation of noncovalently bound inclusion complexes between $\beta$-cyclodextrin and peptides that contain aromatic acid residues was confirmed by electrospray ionization mass spectrometry (ESI-MS) [7]. This work was soon followed by the ESI-MS detection of the $\beta$-cyclodextrin inclusion complex with the drugs, piroxicam and terfenadine [8]. Recently the complexation and chiral selectivity of hydroxypropylsubstituted $\beta$-cyclodextrin derivatives with "guest" molecules was investigated by ESI [9].

In all three studies pertinent to the $\beta$-cyclodextrin complexes, ions that correspond to the expected noncovalent complexes were detected. Much in line with many of the noncovalent studies by ESI, the complexes were known to exist in solution and their corresponding ion in the mass spectrum served to validate the ability to detect them by ESI. In the three ESI studies that reported the detection of inclusion complexes, all the guest molecules that were studied had an aromatic moiety. As noted in the preceding text, the reason for this selection of potential guest molecules is presumably the known complexing affinity of cyclodextrins for aromatic functionalities in aqueous solution. Closer 
a

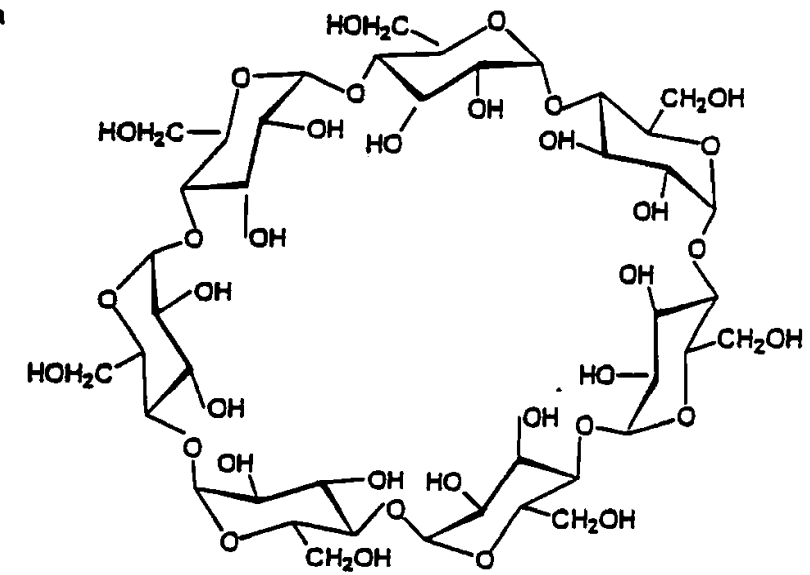

b

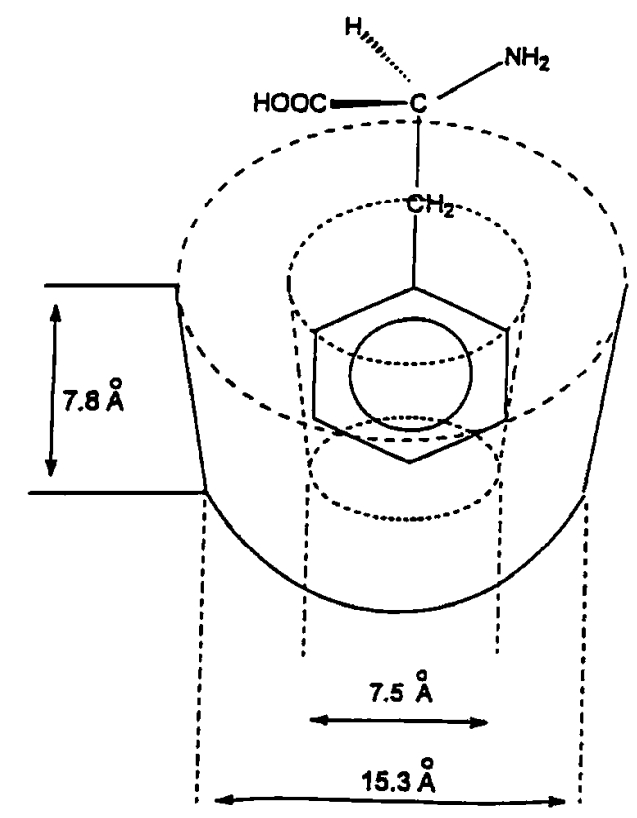

Figure 1. (a) Structure of $\beta$-cyclodextrin. (b) Model of the phenylalanine- $\beta$-cyclodextrin inclusion complex.

examination of all the studies pertinent to host-guest complexation of cyclodextrins reveals that all the guest molecules had, in addition to an aromatic functionality, one other common denominator: all the guest molecules were amines. Unfortunately, a control analysis in which mixtures of $\beta$-cyclodextrin and compounds with aromatic moieties, and without amino groups, as well as mixtures of $\beta$-cyclodextrin compounds that contained amino groups but without aromatic moieties was not performed. Such mixtures constitute the necessary control that will determine whether the observed complex ions are in fact indicative of the "soft" desorption of relatively fragile noncovalently bound solution constituents or are merely electrostatic adducts possibly created during the ESI process.

Along these lines, we discuss here the analysis of mixtures of $\beta$-cyclodextrin with the following four groups of compounds: (1) amino acids that contain aromatic side chains, (2) amino acids that contain nonaromatic basic side chains, (3) peptides that either contain or do not contain an aromatic amino acid, and (4) the hydrophobic compounds, hexane and toluene. The results demonstrate that noncovalent complex ions are evident whether hydrophobic side chains are present or not. The results also suggest that the complexes detected by ESI are probably of an electrostatic nature rather than of a hydrophobic inclusion nature and that they are formed during the electrospray process.

\section{Experimental}

\section{Mass Spectrometer and Interface}

A Finnigan MAT TSQ700 (Finnigan, San Jose, CA) triple quadrupole mass spectrometer equipped with an electrospray ionization source was used. The stainless steel needle supplied with the instrument was replaced with a polyimide-coated fused silica capillary through which solutions were continuously infused. The interface used a coaxial methanol liquid sheath, as well as a coaxial gas sheath. The electrospray needle was maintained at $4500 \mathrm{~V}$ and operated in the positive ion mode. Variations of more than $500 \mathrm{~V}$ had little or no effect on the ion spectral pattern. This was determined by noting that no significant change in relative ion intensities was observed when the the ESI voltage was set at $4000 \mathrm{~V}$ or $5000 \mathrm{~V}$.

\section{Solutions}

$\beta$-Cyclodextrin-amino acid and $\beta$-cyclodextrin-dipeptide solutions. Compounds were dissolved in a solvent mixture made up of water saturated with $\beta$-cyclodextrin (approx. $0.185 \mathrm{mg} / \mathrm{mL} ; 1.6 \times 10^{-4} \mathrm{M}$ ), methanol, and $5 \% \mathrm{v} / \mathrm{v}$ aqueous acetic acid in the ratio 2:1:1. Analyte concentration: $1.0 \mathrm{mg} / \mathrm{mL}$ (Phe, $6.0 \times$ $10^{-3} \mathrm{M}$; Tyr, $5.5 \times 10^{-3} \mathrm{M}$; Try, $4.9 \times 10^{-3} \mathrm{M}$; Lys, $6.8 \times 10^{-3} \mathrm{M}$; Arg, $5.7 \times 10^{-3} \mathrm{M}$; His, $6.5 \times 10^{-3} \mathrm{M}$; ProTyr, $3.6 \times 10^{-3} \mathrm{M}$; Ala-Ile, $4.9 \times 10^{-3} \mathrm{M}$; Ala-Glu, $4.6 \times 10^{-3} \mathrm{M}$.

$\beta$-Cyclodextrin-hydrophobic solutions. Two solutions were prepared. A solvent mixture made up of water saturated with $\beta$-cyclodextrin (approx. $0.185 \mathrm{mg} / \mathrm{mL}$ ), methanol, and $5 \% \mathrm{v} / \mathrm{v}$ aqueous acetic acid in the ratio 2:1:1. To this mixture was added an equal volume of cyclohexane or toluene. The mixture was shaken intermittently for $1 \mathrm{~h}$ and permitted to settle briefly. The aqueous portion was immediately sampled for mass spectral analysis. 


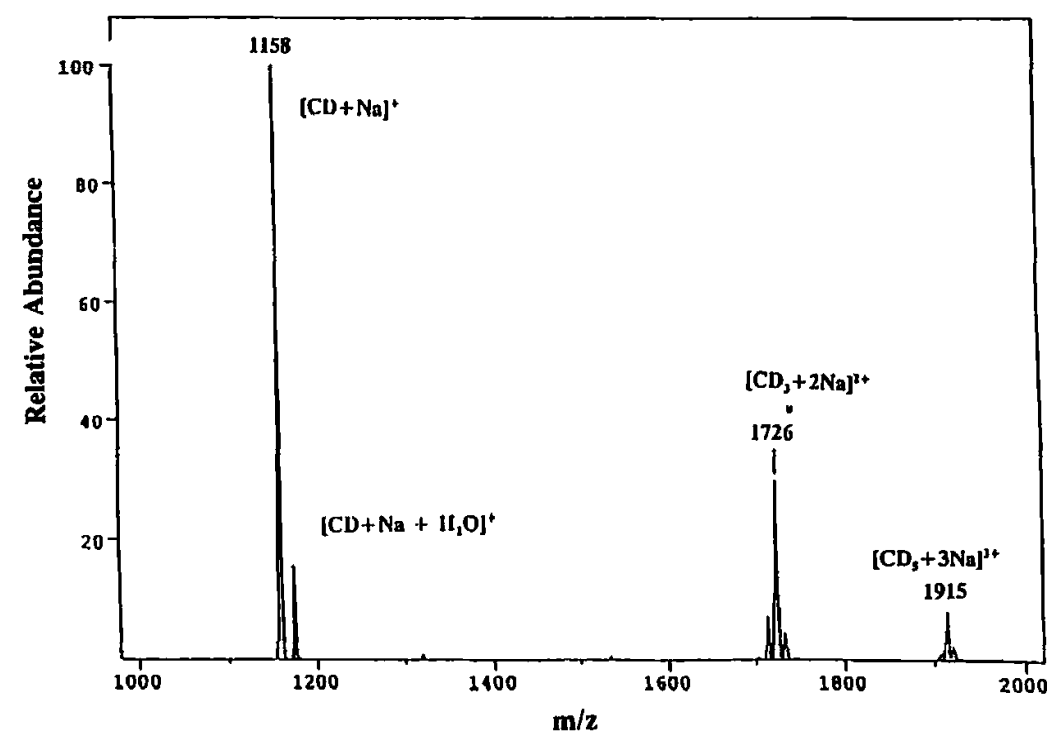

Figure 2. Positive ESI spectra of water saturated with $\beta$-cyclodextrin, methanol, and $5 \% \mathrm{v} / \mathrm{v}$ acetic acid in the ratio $2: 1: 1$.

\section{Results}

\section{$\beta$-Cyclodextrin Solution}

Figure 2 shows the positive ion ESI spectrum acquired from an aqueous solution saturated with $\beta$-cyclodextrin, methanol, and $5 \% \mathrm{v} / \mathrm{v}$ acetic acid in the ratio 2:1:1. Four main adducts are seen, namely, $[\beta-C D+$ $\mathrm{Na}]^{+},\left[\beta-\mathrm{CD}+\mathrm{Na}+\mathrm{H}_{2} \mathrm{O}\right]^{+},\left[(\beta-\mathrm{CD})_{3}+2 \mathrm{Na}\right]^{2+}$, and $\left[(\beta-C D)_{5}+3 \mathrm{Na}\right]^{3+}$. The spectrum is essentially the same as that previously reported, with a noted absence of protonated $\beta$-cyclodextrin in all the studies [7-9]. The lack of protonated species in the acidic media is indicative that $\beta$-cyclodextrin does not contain any readily ionizable groups. The sodium (common laboratory contaminant) adducts are most likely formed from electrostatic binding to the hydroxy groups of $\beta$ cyclodextrin.

\section{Mixtures of $\beta$-Cyclodextrin and Aromatic Amino Acids}

Figure 3 shows the spectra of three solutions that contain a mixture of $\beta$-cyclodextrin and (a) tryptophan, (b) phenylalanine, and (c) tyrosine. As expected, all the $\beta$-cyclodextrin-amino acid complexes are evident. It is interesting to note that a previous study [7] failed to detect the $\beta$-cyclodextrin-tyrosine complex and that, although its absence was somewhat unexpected, this was attributed to the fact that it is the least hydrophobic of the three aromatic amino acids and thus the least likely to form a complex. This seems unlikely because the $\log K$ of the binding of L-tyrosine with $\alpha$-cyclodextrin is midway between those of Lphenylalanine and L-tryptophan, and the equilibrium constants that bind the same guest are similar for both $\alpha$ - and $\beta$-cyclodextrins [10]. We believe that the failure to detect the tyrosine complex in the previous study was, at least in part, due to the following two factors:

1. Tyrosine is the least soluble amino acid in water $(0.5 \mathrm{mg} / \mathrm{mL})$ [11] and the solutions were reportedly made up to concentrations of $1 \mathrm{mg} / \mathrm{mL}$ in a solvent matrix composed of water saturated with $\beta$ cyclodextrin (about $0.185 \mathrm{~g} / \mathrm{L}$ ), methanol, and $5 \%$ aqueous acetic acid in the ratio 2:1:1. (In our own experiments we noted that tyrosine was the only compound that was not completely solubilized in this matrix. The fact that we detected the tyrosine complex at all indicates that some solubility was evident.)

2. The signal-to-noise ratio $(\mathrm{S} / \mathrm{N})$ of the amino acid complexes that were previously [7] reported appears to be only about 5 or 6 . This poor $S / N$, combined with the relative insolubility of the compound, may have contributed to lack of detection.

\section{Mixtures of $\beta$-Cyclodextrin and Nonaromatic Amino Acids}

Figure 4 shows the spectra of three solutions that contain a mixture of $\beta$-cyclodextrin and the three (nonaromatic) basic amino acids: (a) lysine (Lys), (b) arginine (Arg), and (c) histidine (His). Just as in the spectra of Figure 3 , all the protonated $\beta$-cyclodextrin-amino acid complex ions are evident.

\section{Mixtures of $\beta$-Cyclodextrin and Peptides}

Figure 5 shows the spectrum of a solution that contains a mixture of $\beta$-cyclodextrin and the aromatic 

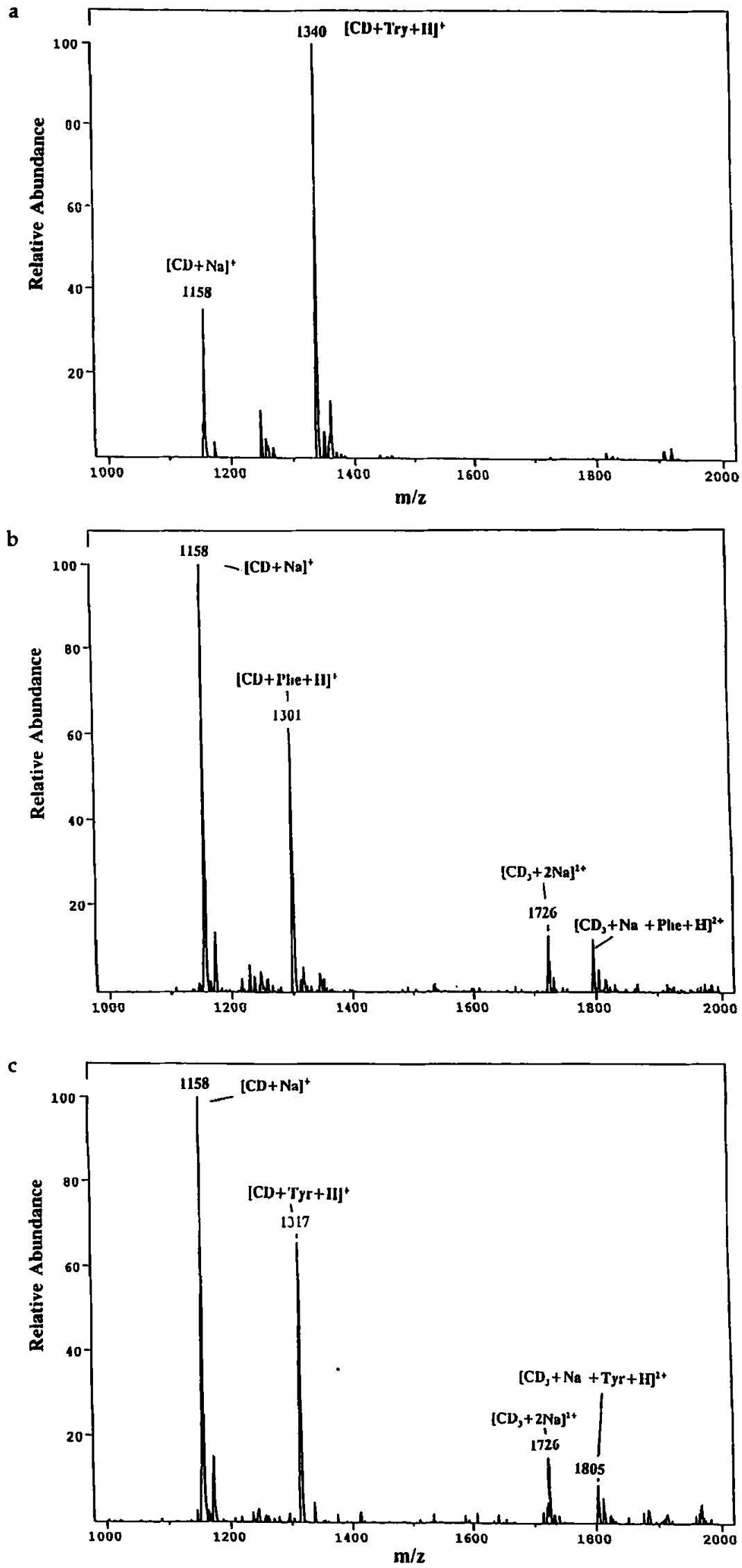

Figure 3. Positive ESI spectra of three aqueous solutions that contain a mixture of $\beta$-cyclodextrin (CD) and (a) tryptophan (Try), (b) phenylalanine (Phe), and (c) tyrosine (Tyr). 
a

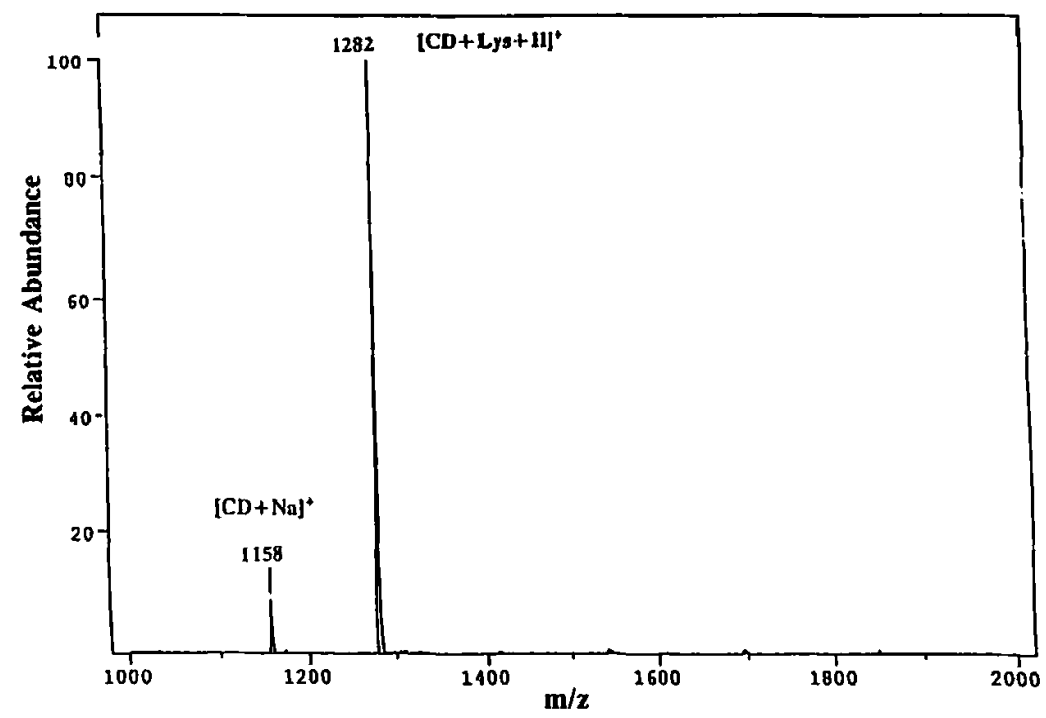

b

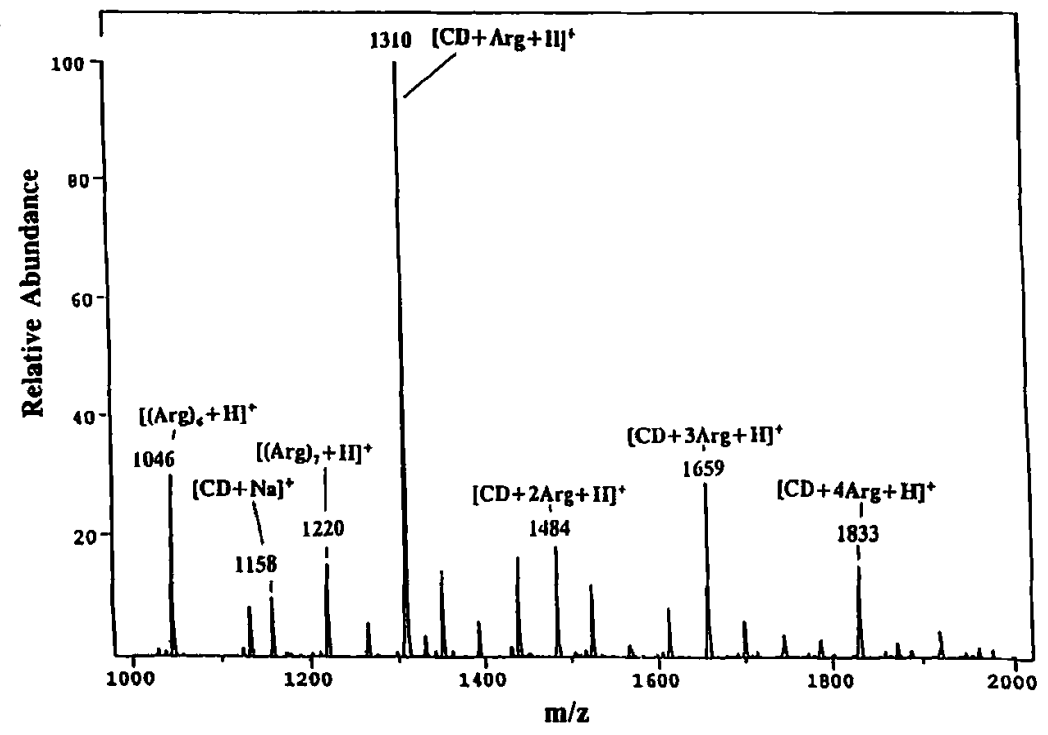

c

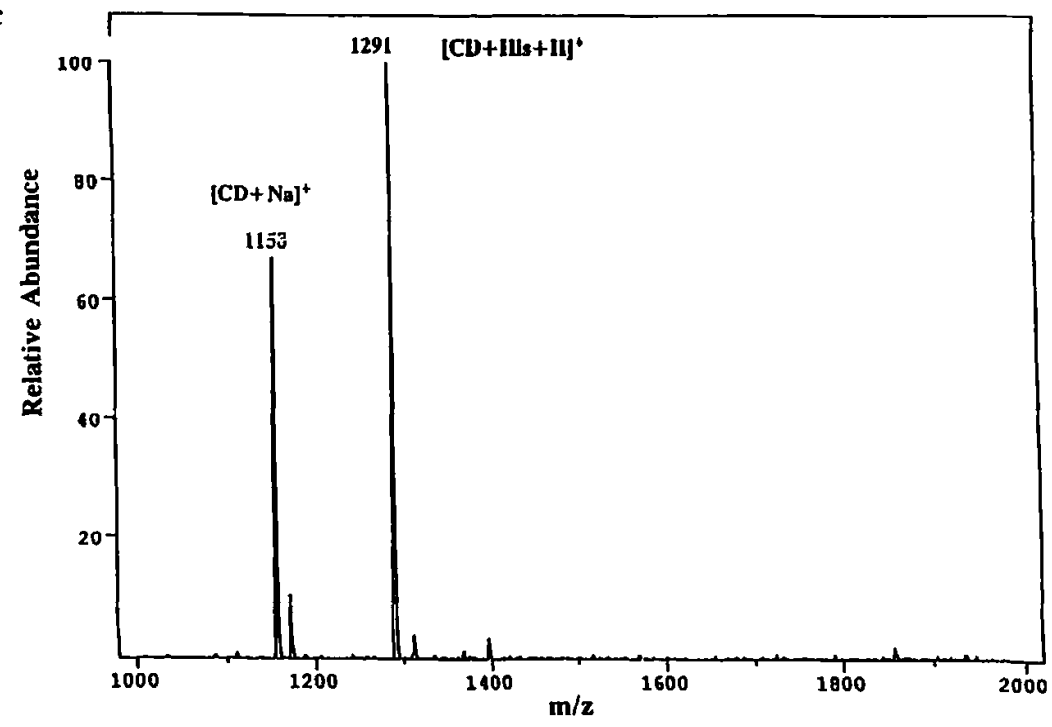

Figure 4. Positive ESI spectra of three aqueous solutions that contain a mixture of $\beta$-cyclodextrin (CD) and (a) lysine (Lys), (b) arginine (Arg) (series of peaks corresponds to multimers of $\left[(\operatorname{Arg})_{x}\right]^{+},\left[C D+(\operatorname{Arg})_{x}\right]^{+}$, etc.), and (c) histadine (His). 
a
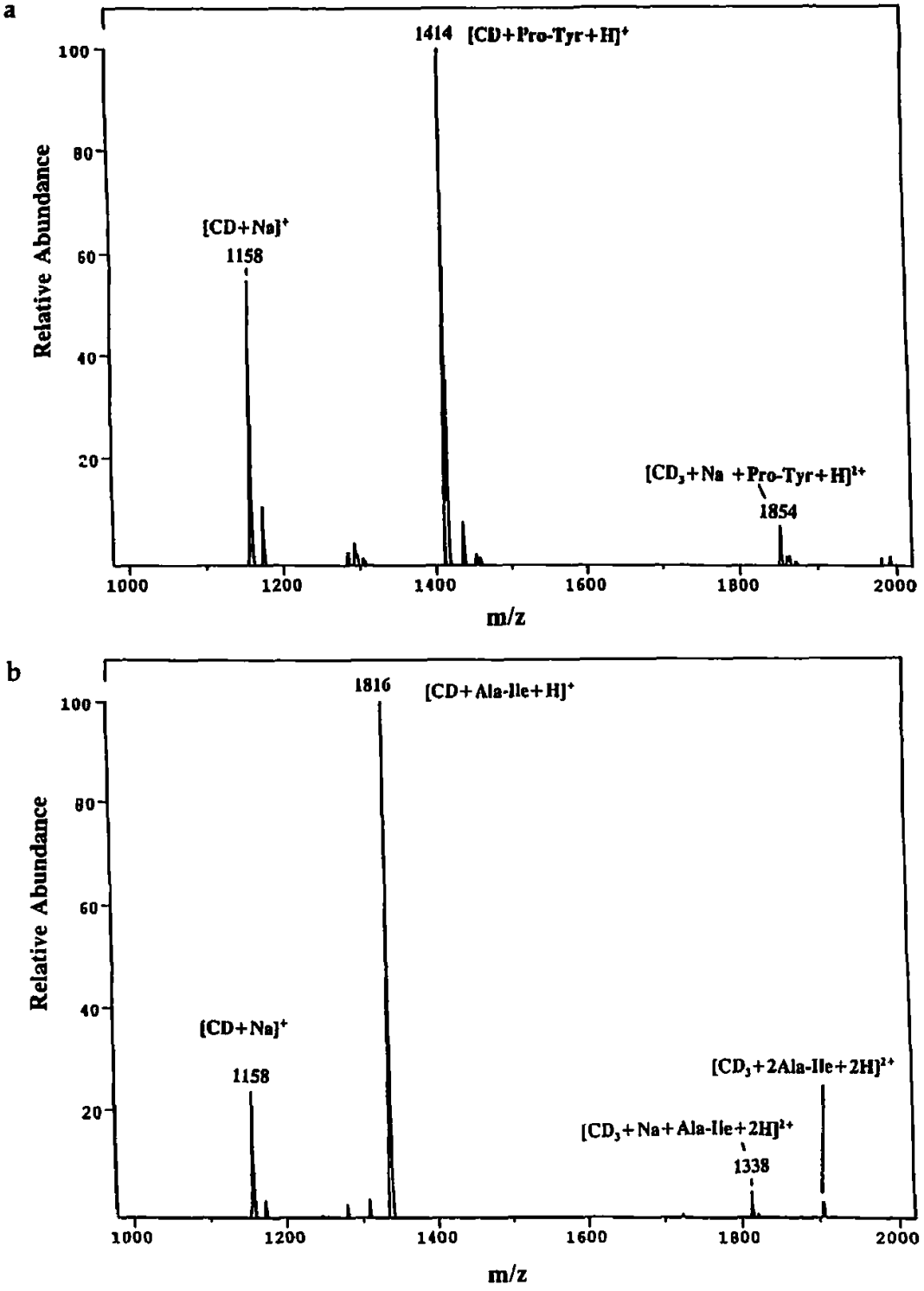

c

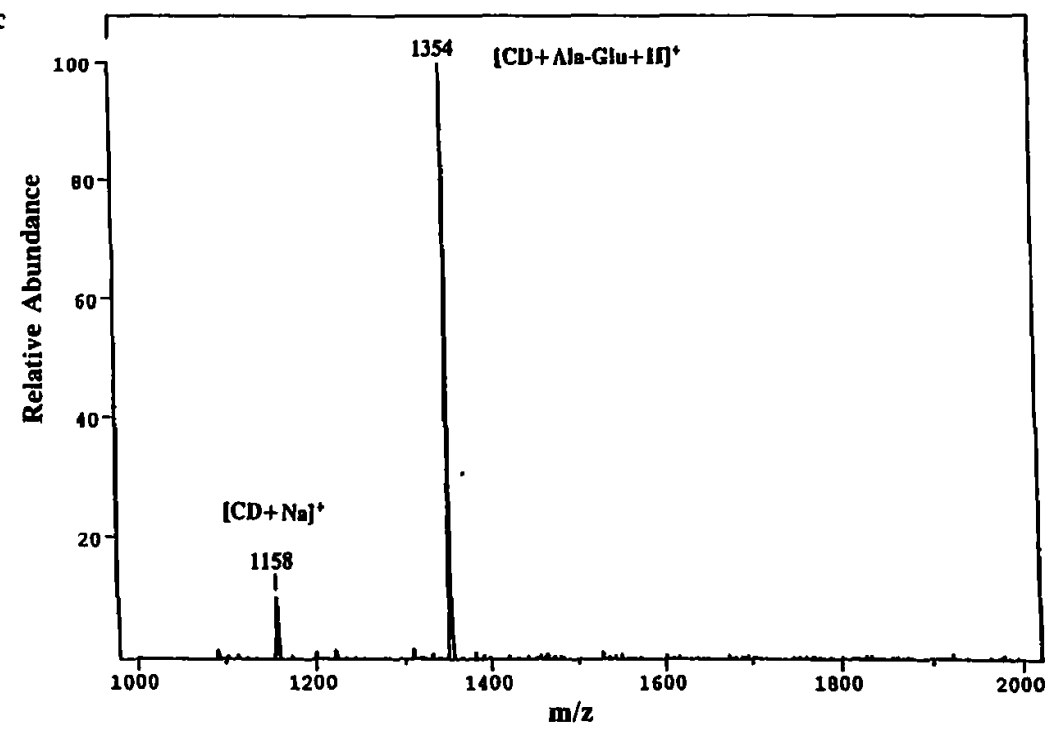

Figure 5. Positive ESI spectra of three aqueous solutions that contain a mixture of $\beta$-cyclodextrin (CD) and (a) proline-tyrosine (Pro-Tyr), (b) alanine-isoleucine (Ala-Ile), and (c) alanineglutamine (Ala-Glu). 
dipeptide Pro-Tyr, as well as the spectra of solutions that contain $\beta$-cyclodextrin and two nonaromatic dipeptides, Ala-Iso and Ala-Glu. The protonated $\beta$ cyclodextrin-dipeptide complex ion is evident from all three solutions, whether an aromatic side chain is present or not.

\section{Mixtures of $\beta$-Cyclodextrin and Hydrophobic Compounds}

Figure 6 shows the spectra of two aqueous solutions after mechanical mixing with cyclohexane (Figure 6a) and toluene (Figure 6b). Neither the $\beta$-cyclodextrin-cyclohexane nor the $\beta$-cyclodextrintoluene complex ion is evident in either spectrum, although the sodiated $\beta$-cyclodextrin is clearly present. Because of the poor solubility of nonpolar compounds in water, it may be concluded that an inclusion complex does not exist in the aqueous solution. On the other hand, aqueous cyclodextrin solutions have been used to remove a variety of organic solvent vapors (including toluene and $n$-hexane) from gases that are brought into contact with the aqueous solution [1]. The aqueous cyclodextrin solutions form inclusion complexes with the organic solvent and have been utilized in much the same way in which activated carbon is used for the removal of harmful solvent vapors from effluents [1]. Finally, previous studies have shown the formation of high concentrations of the complex when a variety of aromatic compounds (including benzene and cyclohexane) have been determined after mechanical mixing with water [1].

\section{Discussion}

As can be inferred from the spectra in Figures 2-6, all amino acids, whether they contain aromatic side chains or not, demonstrate significant ions that correspond to complexation with $\beta$-cyclodextrin. (As of yet, we have

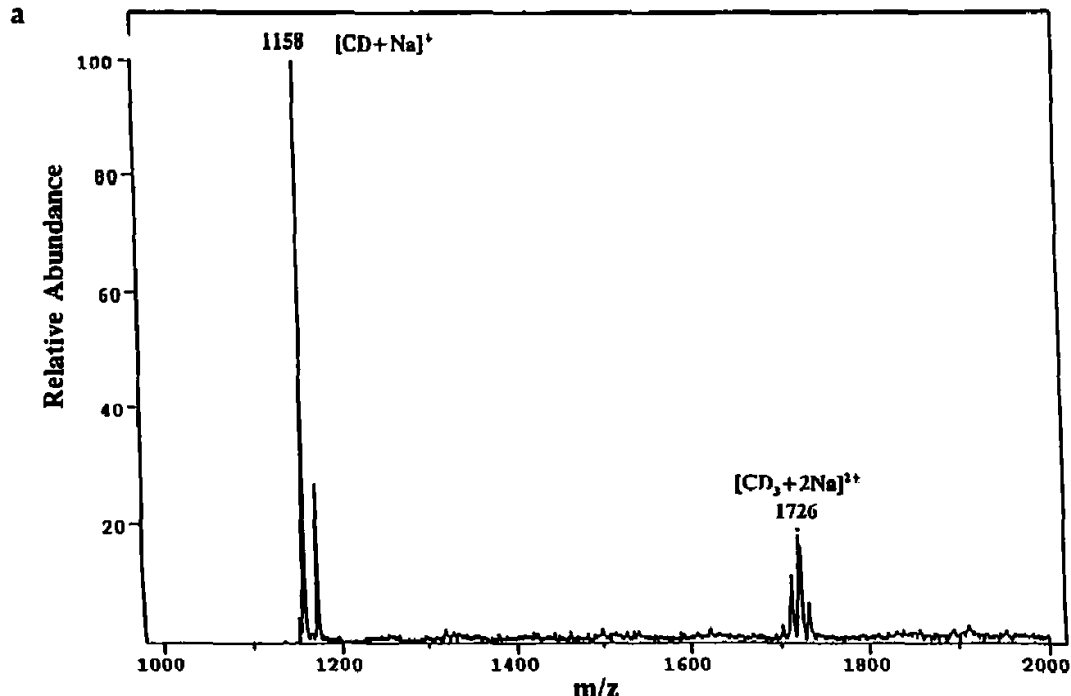

b

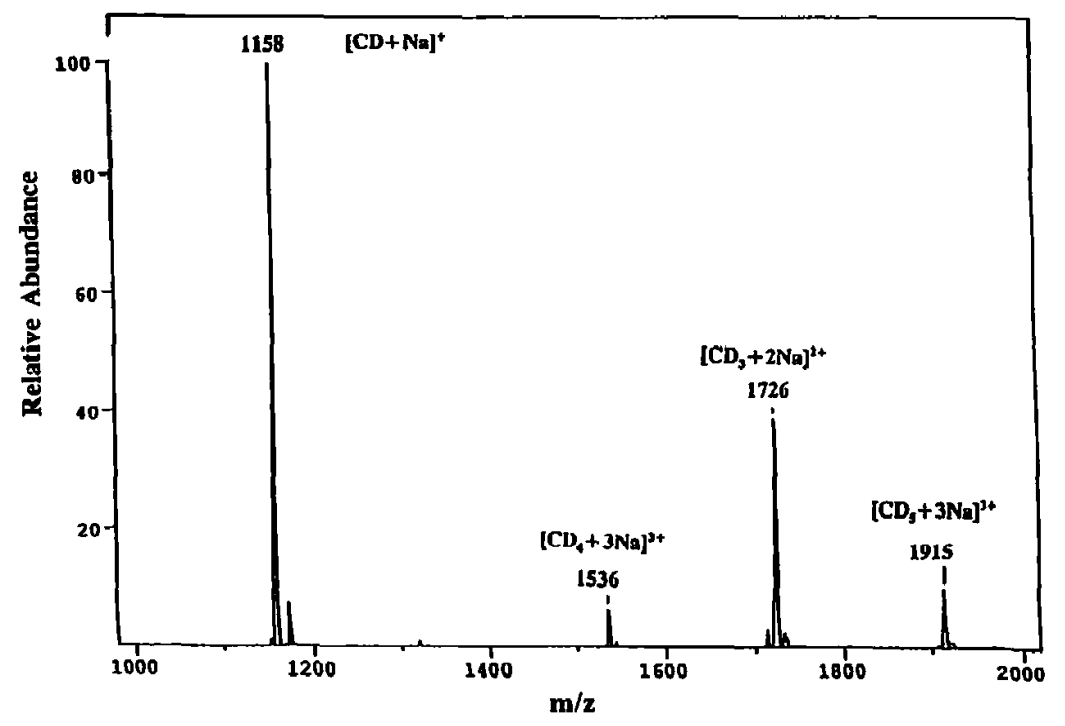

Figure 6. Positive ESI spectra of two solutions that contain an aqueous mixture of $\beta$-cyclodextrin (CD) with (a) cyclohexane and (b) toluene. 
not encountered any amino acid or oligopeptide that does not form a complex ion with $\beta$-cyclodextrin.) For example, prominent $\beta$-cyclodextrin adducts also were observed for the following peptides: Val-Gly-Ser-Glu, Val-Gly-Asp-Glu, Glu-His-Pro-Gly, Arg-Gly-Asp-Ser, Arg-Gly-Glu Ser, Thr-Lys-Pro-Arg, Phe-Leu-Glu-Glu$\mathrm{Val}$, and Phe-Leu-Glu-Glu-Ile.

Even for the most basic amino acids, significant complex ions were observed in the ESI spectra. In fact, when compared to all the other amino acids investigated, it is the basic amino acids that show the greatest complexation with $\beta$-cyclodextrin in the electrospray mass spectra. At this point the question as to whether the basic amino acids form complexes with $\beta$-cyclodextrin in the ambient solution must be addressed. We have conducted an extensive search of the relevant literature, but failed to uncover any explicit data for the nonexistence of a complex with nonaromatic amino acids. This is not surprising because negative results are, after all, usually not published. It is certain nonetheless that only substrates that are less polar than water can form inclusion complexes with cyclodextrins and that the stability of a complex is related to the hydrophobic character and size of the guest molecule. Some fundamental reasons and evidence that imply the absence of complex formation between cyclodextrins and the basic amino acids in ambient solution are elaborated in the following list:

1. Highly hydrophilic molecules complex very weakly or not at all [12].

2. No reaction has been observed with $\mathrm{Na}^{+}$within a $\mathrm{NaCl}$ aqueous solution [1].

3. Although cyclodextrins have been used in the separation of amino acids by micellar electrokinetic capillary chromatography [3] and with the chiral separation of amino acids by capillary zone electrophoresis (CZE) [4], it was a naphthyl group, introduced into the amino acid via dansylation, that was responsible for the complexation with the cyclodextrin.

4. In the analysis of amino acids mixtures that use cyclodextrin supports, only the aromatic amino acids are significantly retained, whereas the rest of the natural amino acids coelute or elute within a very narrow window long before the elution of the aromatic amino acids [13].

5. It has been determined that complex formation is not favored for aromatic compounds that contain amino groups and particularly protonated amino groups [1]. The basic amino acids not only contain amino groups but will likely contain protonated amino groups on their side chains in the acidic media in which they were maintained.

Thus, most present evidence points to the unlikely existence of a solution complex between cyclodextrin and basic amino acids in ambient solution. In view of this, the observation of such protonated complexes in ESI-MS must be attributed to an artifact, that is, a false-positive generated by the ESI process.

The hydrophobic cyclohexane and aromatic toluene solutions demonstrate no complex ion at all, although, as mentioned previously, the degree of solvation for these guests may be in question. The results are readily reproducible.

The absence of the toluene or cyclohexane complex in the ESI spectra is not surprising if we consider the hypothesis that relatively weak hydrophobic bonds cannot survive the electrospray process. On the other hand, it is reasonable to propose that the "complexes" that are observed are the result of the relatively strong electrostatic bonds that arise from interactions of the positively charged ammonium termini of the amino acids with the electronegative oxygen atoms of the $\beta$-cyclodextrin. These interactions are likely to be formed as the solvent is removed. Just as $\mathrm{Na}^{+}$cations will unite with $\mathrm{Cl}^{-}$anions and form a neutral ionic precipitate when water is evaporated from a saturated $\mathrm{NaCl}$ solution in a beaker, analogously, excess analyte cations may form electrostatic bonds with the electronegative oxygens of cyclodextrin as the solvent is removed during the electrospray process. Unlike the example of $\mathrm{NaCl}$, however, electrostatic adducts have a net positive charge and are therefore amenable to detection by the mass spectrometer. Electrostatic interactions are presumably responsible for the generally observed sodium cation adducts. Selva et al. [8] noted that, in the presence of "residual instrumental ammonium ion contamination," the mass spectrum of $\beta$ cyclodextrin showed "practically only the peak at $\mathrm{m} / \mathrm{z}$ $1152\left[\mathrm{CD}+\mathrm{NH}_{4}\right]^{+}, "$ whereas under analogous conditions only the protonated $[\mathrm{M}+\mathrm{H}]^{+}$ions were evident for two aromatic compounds that contained tertiary amino groups. The authors described the differences in cationization $\left(\mathrm{NH}_{4}^{+}\right.$versus $\left.\mathrm{H}^{+}\right)$as "remarkable." We believe that there is a simple explanation for this observation. The observation of the protonated $\beta$ cyclodextrin-aromatic amine complexes (as opposed to ammoniated ions), may in fact have been predicted because $\beta$-cyclodextrin already has demonstrated a strong tendency to form electrostatic complexes with $\mathrm{NH}_{4}^{+}$. Therefore, it is reasonable to assume that any protonated amine (primary, secondary, tertiary) may also form an electrostatic complex with $\beta$-cyclodextrin in the exact same manner that the ammonium ion does. In essence, this class of compounds includes all of the protonated amino acids.

From our observations and the results of past ESI experiments, we predict that, if present at a significant concentration, any protonated compounds that contain amino groups (and probably any positively charged species) will bind to the electronegative oxygen atoms of $\beta$-cyclodextrin (after solvent removal) just as do the sodium and ammonium ions. The "guest" molecules in all the previous reports that "detected the inclusion 
complexes" of $\beta$-cyclodextrin by ESI [7-9] had two things in common: an aromatic moiety and a protonated amino group. Because it was known that aromatic compounds tend to form complexes with $\beta$ cyclodextrin in solution, it was automatically assumed that the occurrence of the ESI ion complex was dictated by the aromatic group. The results presented here suggest that the complexes form during the ESI experiment regardless of whether aromatic groups are present or not. Moreover, given the lack of hexane and toluene complexes with $\beta$-cyclodextrin in the spectra of Figure 6, we are inclined to believe, at least based on the present data, that weak hydrophobic interactions cannot survive in the gas phase or the "soft" ionization of ESI. On the other hand, it may be argued that it is possible that complexes held together by hydrophobic interactions can survive the source conditions, but upon the addition of charge (e.g., addition of $\mathrm{Na}^{+}$in solution or gas phase ), they break up. In other words, the inclusion complex with cylcohexane or toluene may break up after the addition of a charged cation. Because the complex must have a charge to be detected by the mass spectrometer, it is possible that a situation exists that is somewhat similar to the Heisenberg uncertainty principle, that is, to detect the complex we must disturb it in such a way that it breaks up. It is conceivable that if hydrophobic interactions could survive the source conditions, a relatively facile method could be developed whereby electrospray mass spectrometry could be utilized for the detection of highly hydrophobic uncharged species (e.g., CNL scanning, parent scanning, etc., of hydrophobic complex whereby the charged host is detected after collisions).

At this point it is worthwhile to further reflect on the meaning of "soft" as described in the ionization mechanism of ESI. In general it is a relative term compared to other relatively "hard" mass spectrometric ionization techniques such as electron impact, fast atom bombardment, plasma desorption, and even matrix-assisted laser desorption. In essence the term indicates that fragmentation is not as evident from the specific ionization process as it is in the other ionization techniques. The term "soft," however, should not be oversold. In-source fragmentation is after all occasionally observed by ESI. Furthermore, the ESI process is, after all, "hard" enough to eliminate all the waters of hydration that surround some of the largest proteins, albeit in a stepwise fashion. Hundreds or maybe even thousands of water molecules, each exhibiting relatively strong hydrogen bonds to proteins, are "stripped" from the molecule (endothermic process), which results in spectra that are typically dominated by highly charged "water-free" ions. It is, in fact, fortunate that the technique is "hard" enough to facilitate the removal of all the waters of hydration because otherwise the spectra would be exceedingly complex.

Because complete desolvation occurs, it seems likely that noncovalent forces that are weaker than those of at least single water hydration energies cannot with- stand the conditions evident during the electrospray ionization. Relatively strong electrostatic forces, however, do appear to be able to withstand the ESI conditions. It also should be stressed that it is the strength of the noncovalent bond in the gas phase that will determine whether the complex can survive the flight from the source to the detector, not the solution equilibrium constant. This is an especially important point because the mass spectrometer does after all only "observe" gas-phase complexes. There often does appear to be a misconception that only complexes with very high solution formation constants can be detected by ESI. We believe that this misconception arises primarily from the observation that virtually all investigations and reports on the detection of noncovalent species by ESI involve complexes with large formation constants (e.g., $\beta$-cyclodextrin inclusion complexes). A particularly striking example of the fact that the solution formation constant does not determine whether a noncovalent complex will be observed by ESI is evident in the lack of complex ions of an intact hemoglobin tetramer. Likewise, the electrospray analysis of the avidin tetramer generates a spectrum characteristic of the monomeric species [14]. It is possible that because of the limited mass range of most available electrospray instruments, the mass-to-charge ratio of these compounds in their native charge states is not detectable. For example, recently it was reported that both an avidin tetramer and an avidin-biotin complex (solution $K_{D} \sim 10^{-15}$ ) were observed by ESI [15], but their detection required a specially adapted high mass range mass spectrometer.

For the most part, reports of the detection of noncovalent compounds by ESI involve noncovalent complexes that have been studied extensively by alternative means. This is a logical starting point for the determination of the utility of ESI for the characterization of solution-derived noncovalent complexes. There is generally little doubt that the complexes do, in fact, exist in solution. The detection of the complex ion by ESI substantiates something that was previously known. When complexes known to exist in solution do not exhibit a corresponding ion in the ESI spectrum, the results are considered "false-negatives." The true potential benefit of ESI detection of noncovalent complexes will be realized when the method is used for the detection of previously unknown solution-derived complexes. Because anomalous aggregation is so evident from the ESI process, however, for any noncovalent ESI spectrum to be meaningful it must be determined whether the "complexes" detected are in fact not anomalous. As in other disciplines, and as the current study attempts to do, this is readily accomplished by a systematic study of the incidence of false-positives. The utility of the method will be inversely proportional to the prevalence of falsepositives.

It should be pointed out here that we do not argue that all reports on the detection of solution-derived 
noncovalent complexes in fact detect anomalous aggregates. Rather, we encourage that systematic studies be performed to determine the prevalence of falsepositives for each class of noncovalent complexes detected by ESI. Often the determination that observed noncovalent complex ions are indicative of solutionderived complexes rather than the result of anomalous aggregation is made from negative data. For example, the complex may disappear upon denaturation of the host [5], or the complex disappears when replaced with another host $[5,6,16]$, or the complex disappears when the $\mathrm{pH}$ is lowered [17], or the complex disappears when the sample is desalted [18]. Although these negative data are supportive of the intended thesis, they may not necessarily be conclusive. It is possible that by changing solution constituents, the conditions, and/or mechanisms by which anomalous aggregates are formed may be disrupted. The lack of detection of a complex ion with one analyte or the disappearance of a complex after changing the acid concentration by several orders of magnitude does not constitute a control group. A more meaningful control would be substitution of the "guest" molecule with several compounds that resemble the guest in every manner possible with one notable exception; they do not not form complexes with the "host" in solution. For most enzyme-substrate complexes, the enzyme is extremely selective in the substrate with which it will bind. If the mass spectrometer is truly detecting an enzyme-substrate complex, then complexes will not be detected with any of the substituted compounds from the control group. As with any experiment, the larger the control group is, the more convincing the argument will be. This is the control used in the current report and it indicates that what was previously believed to be the detection of an inclusion complex is simply the detection of an anomalous aggregate.

A recent report discussed the dimer formation of oligonucleotides by ESI and included controls in which oligonucleotides that do not form strong base pair complexes were compared to complementary oligonucleotides that do form strong base pair complexes [19]. It should be noted that the forces that hold together complementary strands of oligonucleotides will presumably be stronger than the relatively weak van der Waals forces involved with inclusion complexes. The authors noted that there was only some degree of specificity (as noted from the ESI spectrum) for the detection of complementary base complexes as opposed to noncomplementary bases (for hexamers). The authors further noted that this was somewhat surprising because no stable solution interaction was predicted (from the melting temperature) of the complementary strands in solution at room temperature. The small degree of specificity was attributed possibly to "the capturing of very transient solution-phase complexes as gas-phase ions, and, once formed in the gas phase in the absence of solvent, the duplexes have less tendency to dissociate than if they remained in solution."

It should be noted that the foregoing study, much like our current paper, attempted to determine the incidence of false-positives by choosing as a proper control group, compounds that do not form complexes in solution. This study, like our own, found a large degree of false-positives. In this study and our own, the significance of detection of complex ions known to exist in solution by ESI is greatly compromised by the prevalence of "false-positives" within the control groups.

\section{Conclusion}

The data presented here show that $\beta$-cyclodextrinamino acid complex ions detected by ESI are not limited to aromatic amino acids or peptides that contain aromatic moieties as previously reported. The data further suggest that $\beta$-cyclodextrin-amino acid complexes detected by ESI-MS may not be inclusion complexes, but rather electrostatic adducts. Finally, it is suggested that to determine whether observed complex ions are anomalous adducts or solution-originating complexes, a control study should consist of substitution of the "guest" molecule with a wide range of compounds that resemble the guest in every manner possible with one notable exception: they do not form complexes with the "host" in solution.

\section{Acknowledgments}

We are grateful for support from the Perkin-Elmer Corporation who sponsored this work through the Perkin-Elmer Fellowship.

\section{References}

1. Szejtli, J. Cyclodextrins and their Inclusion Complexes, Akademiai Kiado: Budapest, Hungary, 1982.

2. Szejtli, J. Pharmacol. Tech. Int. 1991, 3, 16-20.

3. Okafo, G. N.; Camilleri, P. J. Microcol. Sep. 1993, 5, 149.

4. Werner, A.; Nassauer, T.; Kiechle, P.; Erni, F. J. Chromatogr. A 1994, 666, 375-379.

5. Ganem, B.; Li, Y.; Henion, J. D. J. Am. Chem. Soc. 1991, 113, 6294-6296.

6. Ganem, B.; Li, Y.; Henion, J. D. J. Am. Chem. Soc. 1991, 113, 7818-7819.

7. Camilleri, P.; Haskins, N. J.; New, A. P.; Saunders, M. R. Rapid Colnmun. Mass Specirom. 1993, 7, 949-952.

8. Selva, A.; Redenti, E.; Zanol, M.; Ventura, P.; Caseta, B. Org. Mass Spectrom. 1993, 28, 983-986.

9. Haskins, N. J.; Saunders, M. R.; Camilleri, P. Rapid Commun. Mass Spectrom. 1994, 8, 423-426.

10. Lewis, E. A.; Hansen, L. D. J. Chem. Soc., Perkin Trans. 2 1973, 2081-2085.

11. CRC Handboak; CRC: Boca Raton, FL, 1992; p 71.

12. Li, S.; Purdy, W. C. Chem. Rev. 1992, 92, 1457-1470. 
13. Szejtli, J.; Zsadon, B.; Cserhati, T. In Ordered Media in Chemical Separations; Hinze, W. L.; Armstrong, D. W., Eds.; American Chemical Society: Washington DC, 1987; Chap. 11.

14. Smith, P. B.; Snyder, A. P.; Simmons, T. A.; Vestling, M. M. Presented at the 41st ASMS Conference On Mass Spectrometry and Allied Topics, San Francisco, CA, May 30-June 4, 1993; $p$ 76a.

15. Schwartz, B. L.; Light-Wahl, K.; Smith, R. D. J. Am. Soc. Mass Spectrom. 1994, 5, 201-204.
16. Hsieh, Y. L.; Li, Y,; Henion, J. D.; Ganem, B. Biol. Mass Spectrom. 1994, 23, 272-276.

17. Camilleri, P.; Haskins, N. J. Rapid Commun. Mass Spectrom. 1993, 7, 603-604.

18. Goodlett, D. R.; Camp, D. G.; Hardin, C. C.; Corregan, M.; Smith, R. D. Biol. Mass Spectrom. 1993, 22, 181-183.

19. Ding, J.; Anderegg, R. J. Presented at the 42nd ASMS Conference On Mass Spectrometry and Allied Topics, Chicago, 1994; $\mathrm{p} 913$. 